\title{
Early experience with delamanid- containing regimens in the treatment of complicated multidrug-resistant tuberculosis in Hong Kong
}

To the Editor:

Hong Kong is an intermediate tuberculosis (TB) burden region with a disease notification rate of 60.5 per 100000 population in 2015. With the use of supervised treatment since 1970s and the use of drug susceptibility testing (DST) for guiding use of TB drugs for several decades [1], TB drug resistance rates in Hong Kong have declined and the multidrug-resistant (MDR)-TB rate has been kept at $\sim 1 \%$. Successful control of MDR-TB was partly attributable to judicious use of ofloxacin and later levofloxacin in the 1990s [2, 3], the introduction of linezolid in the 2000s [4] among patients with fluoroquinolone-resistant MDR-TB or extensively drug-resistant (XDR)-TB, with intermittent dosing to enable its prolonged use [5], and selective use of delamanid among patients with complicated MDR-TB since 2012. To echo our support for using delamanid in the treatment of MDR-TB [6, 7], we would like to report our early experience in Hong Kong regarding the use of delamanid-containing regimens among patients with pre-XDR-TB (MDR-TB with bacillary resistance to either fluoroquinolone or second-line injectable agents) or XDR-TB.

Since 2012, we have used delamanid in a total of 11 patients with pre-XDR-TB or XDR-TB. Table 1 summarises their clinical profiles. There were seven females, aged from 29 to 59 years (median 48 years), and four males, aged from 44 to 59 years (median 52.5 years). Except for one Pakistani, all were Chinese. Comorbidity was common, with four having diabetes mellitus and one having dermatomyositis treated with chronic systemic corticosteroids. All patients had HIV status checked and none was infected by HIV. All received high-dose levofloxacin throughout, predominantly $750 \mathrm{mg}$ daily. Except for two patients (patients 2 and 3) with discontinuation of linezolid after 5.5 months and 0.5 month, respectively, owing to adverse events, all received the oxazolidinone for a prolonged period. A total of eight patients (excluding patients 1-3) had delamanid and linezolid initiated concurrently. Patient 1 had delamanid and high-dose isoniazid added to a linezolid-containing regimen when treatment response was poor. Patients 2 and 3 were given delamanid to substitute for linezolid owing to linezolid intolerance, when treatment response was satisfactory.

Treatment outcomes regarding use of delamanid-containing regimens have been promising. Except for patient 1, all achieved early sputum culture conversion within 3 months dafter starting MDR-TB treatment. Nine were cured after a median treatment duration of 13.0 months (range 12.0-27.0 months) and not found to have relapse after a median follow-up period of 390 days (range 0-720 days). One with good progress is still receiving treatment. Treatment was prolonged beyond 20 months in three patients (patients 2, 3 and 11) owing to concerns about an increase in relapse risk due to poorly controlled diabetes mellitus. Treatment failure occurred in patient 1, who had fluoroquinolone-resistant MDR-TB with I572F mutation (located outside $r p o B$ hotspot), low-level isoniazid resistance, bacillary resistance to ethionamide and dermatomyositis treated with chronic systemic corticosteroids. Patient 1 was initially treated using a linezolid-containing regimen that somehow omitted concurrent use of high-dose isoniazid. When treatment response to the linezolid-containing regimen was poor, high-dose isoniazid and delamanid were added, but treatment response was unsatisfactory. I572F mutation made it difficult to

@ERSpublications

It is probably safe to give delamanid beyond 6 months, with once-daily dosing after the first 1-2 months http://ow.ly/zoCv30jyCj0

Cite this article as: Chang K-C, Leung EC-C, Law W-S, et al. Early experience with delamanid-containing regimens in the treatment of complicated multidrug-resistant tuberculosis in Hong Kong. Eur Respir J 2018; 51: 1800159 [https://doi.org/10.1183/13993003.00159-2018]. 
TABLE 1 Clinical profiles of a cohort of 11 patients with complicated multidrug-resistant (MDR) tuberculosis (TB) who were given delamanid (DIm)

\begin{tabular}{|c|c|c|c|c|c|c|c|c|c|c|c|c|}
\hline Patient & $\begin{array}{l}\text { Starting } \\
\text { MDR-TB } \\
\text { treatment }\end{array}$ & $\begin{array}{l}\text { Sex/ } \\
\text { age } \\
\text { years }\end{array}$ & Comorbidity & $\begin{array}{l}\text { Drugs with } \\
\text { bacillary } \\
\text { resistance }\end{array}$ & $\begin{array}{l}\text { Past TB } \\
\text { treatment }\end{array}$ & $\begin{array}{l}\text { Total } \\
\text { treatment } \\
\text { duration } \\
\text { months }\end{array}$ & $\begin{array}{l}\text { Dlm } \\
\text { treatment } \\
\text { duration } \\
\text { months }\end{array}$ & $\begin{array}{l}\text { Lzd } \\
\text { treatment } \\
\text { duration } \\
\text { months }\end{array}$ & $\begin{array}{c}\text { Other drugs } \\
\geqslant 1 \text { month }\end{array}$ & $\begin{array}{c}\text { QTc } \\
\text { range } \\
\text { ms }\end{array}$ & $\begin{array}{l}\text { Time to culture } \\
\text { conversion after } \\
\text { starting MDR-TB } \\
\text { treatment days }\end{array}$ & $\begin{array}{l}\text { Outcome (follow-up } \\
\text { duration after } \\
\text { completing MDR-TB } \\
\text { treatment }^{\text {"1 }} \text { days) }\end{array}$ \\
\hline 1 & $\begin{array}{c}\text { December } \\
2012\end{array}$ & $M / 57$ & DMS on CS & $\begin{array}{c}\mathrm{S}, \mathrm{H}^{\mathrm{r}}, \mathrm{E}, \mathrm{Z}, \mathrm{Ofx}, \\
\text { Eto }\end{array}$ & Yes & 35.0 & 12.5 & 29.5 & $\begin{array}{l}\mathrm{Lfx}^{+}, \mathrm{Km}^{+}, \mathrm{H}^{\mathrm{h}+} \\
\text { Pto, E, PAS, Cs }\end{array}$ & $408-443$ & NA & Failure \\
\hline 2 & June 2014 & $\mathrm{~F} / 48$ & DM & $H^{r}, R, Z$, Lfx, Eto & Yes & 27.0 & 6.0 & 5.5 & $\begin{array}{l}\mathrm{Lfx}^{+}, \mathrm{Km}^{+}, \mathrm{H}^{\mathrm{h}+} \\
\text { Pto, Z, E, PAS, } \\
\text { Cs }\end{array}$ & $453-467$ & 91 & Cured (390) \\
\hline 3 & $\begin{array}{c}\text { September } \\
2014\end{array}$ & $\mathrm{~F} / 57$ & DM & $\begin{array}{c}S, H, R, E, L f x \\
M f x\end{array}$ & No & 21.0 & 9.0 & 0.5 & $\begin{array}{c}\mathrm{Lfx}^{+}, \mathrm{Am}^{+}, \mathrm{Pto}^{+} \\
\text {Z, PAS, Cs }\end{array}$ & $435-452$ & 17 & Cured (461) \\
\hline $4^{\S}$ & $\begin{array}{c}\text { November } \\
2014\end{array}$ & $\mathrm{~F} / 54$ & & $\begin{array}{c}\mathrm{S}, \mathrm{H}^{\mathrm{r}}, \mathrm{R}, \mathrm{E}, \mathrm{Z} \\
\mathrm{Lfx}, \mathrm{Am}, \mathrm{Km}, \\
\mathrm{Cm}, \mathrm{PAS}\end{array}$ & Yes & 12.0 & 12.0 & 12.0 & $\mathrm{Lfx}^{+}, \mathrm{H}^{\mathrm{h+}}$ & $410-436$ & 0 & Cured (720) \\
\hline $5^{\S}$ & March 2015 & $F / 48$ & & $\begin{array}{c}\mathrm{S}, \mathrm{H}, \mathrm{R}, \mathrm{Z}, \mathrm{Am}, \\
\mathrm{Km}, \mathrm{Cm}\end{array}$ & Yes & 13.0 & 6.0 & 13.0 & $\mathrm{Lfx}^{+}, \mathrm{E}$ & $429-440$ & 14 & Cured (672) \\
\hline $6^{\S}$ & April 2015 & $M / 44$ & $\begin{array}{c}\text { Alcoholic } \\
\text { liver disease }\end{array}$ & $H, E, Z, L f x$ & Yes & 13.0 & 12.5 & 13.0 & $\mathrm{Lfx}^{+}, \mathrm{Am}^{+}, \mathrm{PAS}$ & $432-459$ & 28 & Cured (454) \\
\hline $7^{\S}$ & $\begin{array}{l}\text { September } \\
2015\end{array}$ & $M / 48$ & & $\begin{array}{c}\mathrm{S}, \mathrm{H}^{\mathrm{r}}, \mathrm{R}, \mathrm{E}, \mathrm{Z} \\
\mathrm{Am}, \mathrm{Km}, \mathrm{Cm}, \\
\mathrm{Lfx}\end{array}$ & No & 13.0 & 12.0 & 11.5 & $\mathrm{Lfx}^{+}, \mathrm{H}^{\mathrm{h}+}, \mathrm{PAS}$ & $389-415$ & 67 & Cured (338) \\
\hline $8^{\S}$ & June 2016 & $F / 29$ & & $\begin{array}{l}\mathrm{S}, \mathrm{H}, \mathrm{R}, \mathrm{E}, \mathrm{Z} \\
\mathrm{Am}, \mathrm{Km}, \mathrm{Cm} \text {, } \\
\text { Lfx, Mfx, Eto, } \\
\text { PAS, Cs }\end{array}$ & No & 14.5 & 12.0 & 14.5 & $\begin{array}{l}\text { Lfx', Pto, Z, } \\
\text { PAS, Cs }\end{array}$ & $401-471$ & 79 & Cured (174) \\
\hline $9^{\S}$ & $\begin{array}{c}\text { December } \\
2016\end{array}$ & $F / 59$ & DM & $\begin{array}{l}\text { S, H, R, E, Z, } \\
\text { Lfx, Eto, Am, } \\
\text { Km, Cm }\end{array}$ & Yes & $>14.5$ & $>14.5$ & $>14.5$ & $\mathrm{Lfx}^{+}, \mathrm{PAS}, \mathrm{Cs}$ & $423-446$ & 58 & $\begin{array}{l}\text { Treatment in } \\
\text { progress }\end{array}$ \\
\hline $10^{\S}$ & $\begin{array}{c}\text { December } \\
2016\end{array}$ & $F / 29$ & & $\begin{array}{l}\mathrm{S}, \mathrm{H}, \mathrm{R}, \mathrm{E}, \mathrm{Z} \\
\mathrm{Am}, \mathrm{Km}, \mathrm{Cm}\end{array}$ & No & 13.0 & 6.5 & 13.0 & $\mathrm{Lfx}^{+}, \mathrm{PAS}, \mathrm{Cs}$ & $418-486$ & 35 & Cured (0) \\
\hline $11^{\S}$ & April 2016 & $M / 59$ & DM & $\begin{array}{c}\text { S, H, R, E, Ofx, } \\
\text { Mfx, Eto, Km, } \\
\text { PAS, Cs }\end{array}$ & No & 22.0 & 22.0 & 22.0 & $\mathrm{Lfx}^{+}, \mathrm{Km}, \mathrm{E}, \mathrm{Cs}$ & $404-447$ & 76 & Cured (0) \\
\hline
\end{tabular}

Lzd: linezolid; QTc: corrected QT interval; M: male; F: female; DMS: dermatomyositis; CS: corticosteroids; S: streptomycin; $\mathrm{H}^{\mathrm{r}}$ : low-level isoniazid resistance; E: ethambutol; Z: pyrazinamide; Ofx: ofloxacin; Eto: ethionamide; Lfx: levofloxacin; Km: kanamycin; $\mathrm{H}^{\mathrm{h}}$ : high-dose isoniazid; Pto: prothionamide; PAS: para-aminosalicylic acid; Cs: cycloserine; NA: not applicable; DM: diabetes mellitus; R: rifampicin; Mfx: moxifloxacin; Am: amikacin; Cm: capreomycin. ": age upon starting MDR-TB treatment. ": follow-up duration after completing treatment was estimated from the duration between the last follow-up date and the treatment completion date. ${ }^{+}$: drugs with likely in vitro activity refer to those to which drug susceptibility testing suggests bacillary susceptibility: however, in the case of fluoroquinolone resistance and $\mathrm{H}^{\mathrm{r}}$, high-dose later-generation fluoroquinolone and $\mathrm{H}^{\mathrm{h}}$ are considered to have likely in vitro activity, respectively, and in the context of pre-extensively drug resistant (XDR)-TB and XDR-TB, owing to poor test reliability, E, PAS and Cs were not considered to have likely in vitro activity even if drug susceptibility testing suggested bacillary susceptibility. ${ }^{\S}$ : Dlm was concurrently initiated with Lzd in these eight patients; in the other patients, Dlm was given later (please refer to the main text). 
monitor drug resistance pattern during treatment as a result of excessively slow growth observed in the culture isolates. Despite the lack of evidence from phenotypic or molecular DST, the probability of sequentially acquired bacillary resistance, first to linezolid and then delamanid, was high. The patient was kept in the hospital throughout MDR-TB treatment until death, which occurred $\sim 6$ months after termination of MDR-TB treatment. Our experience with patient 1 corroborated the relatively frequent $\left(10^{-5}\right.$ to $\left.10^{-6}\right)$ spontaneous mutations that confer resistance to nitroimidazoles such as delamanid and pretomanid. When a delamanid-containing regimen is used to treat fluoroquinolone-resistant MDR-TB, it would probably be better to include drugs with a lower propensity to develop drug resistance, such as linezolid or bedaquiline. The combined use of bedaquiline and delamanid has been reported by at least three case studies [8-10]. Trial 213 suggested that delamanid could help prevent amplification of drug resistance among companion drugs [11]. However, this property should not be confused with the propensity of Mycobacterium tuberculosis to develop resistance against delamanid, which is related to bacillary load, frequency of spontaneous resistance-associated mutations and potency of the treatment regimen.

In Hong Kong, an ambulatory treatment policy is adopted regardless of the drug resistance pattern. To facilitate treatment supervision in the community after initial treatment in the hospital, we gave delamanid $200 \mathrm{mg}$ once daily instead of $100 \mathrm{mg}$ twice daily after the first $1-2$ months. We did not check serum drug levels. Although delamanid exposure for at least the first 2 months may be optimally achieved with $100 \mathrm{mg}$ twice daily [12], delamanid $200 \mathrm{mg}$ once daily is probably efficacious with reference to the published literature regarding the pharmacokinetics and early bactericidal activity [13, 14]. Delamanid has a long plasma half-life of 30-38 h, with a steady-state concentration reached after 10-14 days [13], maximum exposure at the $300 \mathrm{mg}$ dosage (oral administration) [14], and substantial and similar early bactericidal activity over 14 days for $200 \mathrm{mg}$ once daily and $300 \mathrm{mg}$ once daily [14]. Although efficacy findings from Trial 213 suggested small benefit from adding delamanid to an optimised background regimen when $200 \mathrm{mg}$ once daily was used after the first 2 months, this might be related more to an unexpectedly good treatment outcome in the control group than inadequate delamanid exposure, which would be internally inconsistent with another Trial 213 finding that suggested delamanid could help prevent amplification of drug resistance [11]. Our early experience echoes findings from Trial 213 that it would probably be safe and efficacious to give delamanid $200 \mathrm{mg}$ once daily after the first 2 months.

In none of our patients did we find it necessary to stop delamanid owing to prolongation of corrected QT interval, which was periodically monitored throughout treatment. Delamanid was initiated in the hospital to enable close monitoring for about 6-9 weeks. ECG was monitored once weekly in the hospital and once monthly in the chest clinic after hospital discharge. Further delineation of the optimal way to monitor the corrected QT interval under programmatic settings is required.

In the absence of adverse events, use of delamanid was substantially extended beyond 6 months by $\geqslant 3$ months in eight patients ( 9 months in one, $\sim 12$ months in five and $>12$ months in two), with the longest duration of use being 22 months. Extending use of delamanid beyond 6 months, alongside use of linezolid, was found to be well tolerated.

Restricting analysis to eight patients with linezolid and delamanid concurrently initiated in the regimen (excluding patients 1-3), seven patients (patients 4-8, 10 and 11) have been cured after receiving treatment for 12.0-22.0 months (median 13.0 months), and followed up after completing treatment for a median of 338 days (range $0-720$ days). Patient 9 is still receiving treatment with good progress. Patient 11 received treatment for 22 months despite sputum culture conversion within 3 months after starting MDR-TB treatment owing to concerns about a higher relapse risk due to poorly controlled diabetes mellitus. We have been successfully treating the majority of fluoroquinolone-susceptible MDR-TB patients with regimens that lasted for only 12-15 months (1 year after culture conversion) [15], except for those with comorbidity that may substantially increase the relapse risk. Our early experience with delamanid-containing regimens suggests that treatment could be stopped 1 year after sputum culture conversion among difficult MDR-TB patients with delamanid and linezolid concurrently initiated in the treatment regimen, except for those with comorbidity that may substantially increase the relapse risk.

In summary, our early experience suggests that: 1) fluoroquinolone-resistant MDR-TB or XDR-TB may be successfully treated with shorter (about 12-15 months) regimens comprising both delamanid and linezolid, except for those with comorbidity that may substantially increase the relapse risk; 2) it would probably be better to initiate delamanid alongside linezolid; 3) longer courses of delamanid are well tolerated and safe; and 4) delamanid could be given once daily instead of twice daily after the first 1-2 months. Further evaluation in a larger patient database is required to substantiate these preliminary observations. 
Kwok-Chiu Chang ${ }^{1}$, Eric Chung-Ching Leung ${ }^{1}$, Wing-Sze Law ${ }^{1}$, Wai-Man Leung ${ }^{1}$, Lai-Bun Tai ${ }^{1}$, Shuk-Nor Lee ${ }^{1}$, Fai-Man Lam ${ }^{2}$, Chi-Hung Chau ${ }^{2}$, Thomas Yun-Wing Mok ${ }^{3}$, Wing-Wai Yew ${ }^{4}$ and Chi-Chiu Leung ${ }^{1}$

${ }^{1}$ Tuberculosis and Chest Service, Dept of Health, Hong Kong SAR, China. ${ }^{2}$ Tuberculosis and Chest Unit, Grantham Hospital, Hong Kong SAR, China. ${ }^{3}$ Dept of Respiratory Medicine, Kowloon Hospital, Hong Kong SAR, China. ${ }^{4}$ Stanley Ho Centre for Emerging Infectious Diseases, The Chinese University of Hong Kong, Hong Kong SAR, China.

Correspondence: Kwok-Chiu Chang, Tuberculosis and Chest Service, Dept of Health, Wanchai Chest Clinic, 1st Floor, Wanchai Polyclinic, 99, Kennedy Road, Wanchai, Hong Kong SAR, China. E-mail: kc_chang@dh.gov.hk

Received: Jan 242018 | Accepted after revision: March 242018

Conflict of interest: W.W. Yew was a consultant to Otsuka, with an honorarium, until July 2016.

\section{References}

1 Kam KM, Yip CW. Surveillance of Mycobacterium tuberculosis susceptibility to second-line drugs in Hong Kong, 1995-2002, after the implementation of DOTS-plus. Int J Tuberc Lung Dis 2004; 8: 760-766.

2 Yew WW, Chan $\mathrm{CK}$, Chau $\mathrm{CH}$, et al. Outcomes of patients with multidrug-resistant pulmonary tuberculosis treated with ofloxacin/levofloxacin-containing regimens. Chest 2000; 117: 744-751.

3 Yew WW, Chan CK, Leung CC, et al. Comparative roles of levofloxacin and ofloxacin in the treatment of multidrug-resistant tuberculosis: preliminary results of a retrospective study from Hong Kong. Chest 2003; 124: $1476-1481$.

4 Yew WW, Chang KC, Chau $\mathrm{CH}$. What is the optimal dosage of linezolid in treatment of complicated multidrug-resistant tuberculosis? Eur Respir J 2009; 34: 1492-1494.

5 Chang K-C, Yew W-W, Cheung S-W, et al. Can intermittent dosing optimize prolonged linezolid treatment of difficult multidrug-resistant tuberculosis? Antimicrob Agents Chemother 2013; 57: 3445-3449.

6 Hafkin J, Hittel N, Martin A, et al. Early outcomes in MDR-TB and XDR-TB patients treated with delamanid under compassionate use. Eur Respir J 2017; 50: 1700311.

7 Kuksa L, Barkane L, Hittel N, et al. Final treatment outcomes of multidrug- and extensively drug-resistant tuberculosis patients in Latvia receiving delamanid-containing regimens. Eur Respir J 2017; 50: 1701105.

8 Tadolini M, Lingtsang RD, Tiberi S, et al. First case of extensively drug-resistant tuberculosis treated with both delamanid and bedaquiline. Eur Respir J 2016; 48: 935-938.

9 Lachâtre M, Rioux C, Dû DL, et al. Bedaquiline plus delamanid for XDR tuberculosis. Lancet Infect Dis 2016; 16: 294.

10 Maryandyshev A, Pontali E, Tiberi S, et al. Bedaquiline and delamanid combination treatment of 5 patients with pulmonary extensively drug-resistant tuberculosis. Emerging Infect Dis 2017; 23: 1718-1721.

11 World Health Organization. WHO position statement on the use of delamanid for multidrug-resistant tuberculosis. 2018 Jan. Report No.: WHO/CDS/TB/2018.1. Geneva, WHO, 2018.

12 Gupta R, Geiter LJ, Hafkin J, et al. Delamanid and QT prolongation in the treatment of multidrug-resistant tuberculosis. Int J Tuberc Lung Dis 2015; 19: 1261-1262.

13 Ryan NJ, Lo JH. Delamanid: first global approval. Drugs 2014; 74: 1041-1045.

14 Diacon AH, Dawson R, Hanekom M, et al. Early bactericidal activity of delamanid (OPC-67683) in smear-positive pulmonary tuberculosis patients. Int J Tuberc Lung Dis 2011; 15: 949-954.

15 Leung ECC, Yew WW, Leung CC, et al. Shorter treatment duration for selected patients with multidrug-resistant tuberculosis. Eur Respir J 2011; 38: 227-230. 